\title{
Pathogenic Characteristics and Risk Factors for ESKAPE Pathogens Infection in Burn Patients
}

\section{Zhaoyinqian Li* \\ Jingling $\mathrm{Xie}^{*}$ \\ Jiaxin Yang (D) \\ Siyi Liu \\ Zixuan Ding \\ Jingchen Hao \\ Yinhuan Ding \\ Zhangrui Zeng \\ Jinbo Liu (D)}

Department of Laboratory Medicine, The Affiliated Hospital of Southwest Medical University, Luzhou, 646000, Sichuan,

People's Republic of China

*These authors contributed equally to this work
Correspondence: Jinbo Liu Department of Laboratory Medicine, The Affiliated Hospital of Southwest Medical University, Taiping Street, Luzhou, 646000, Sichuan, People's Republic of China Tel +860830 3165730

Email Liulab2019@I63.com
Objective: This study aimed to determine the clinical manifestations, antimicrobial resistance, molecular characteristics, and risk factors for ESKAPE pathogens infection in burn patients.

Methods: A retrospective study of 187 burn patients infected with ESKAPE pathogens was conducted at the Department of Plastic and Burn Surgery of the Affiliated Hospital of Southwest Medical University (Luzhou, China) from October 2018 to June 2021. All strains were identified using a MicroScan WalkAway 96 Plus System, and antimicrobial susceptibilities were determined using the VITEK system or the disk diffusion method. The antimicrobial resistance genes of multi-drug resistant ESKAPE (MDR-ESKAPE) were detected by polymerase chain reaction (PCR). The multivariable logistic regression analysis was used to estimate the risk factors for ESKAPE infection and MDR-ESKAPE infection.

Results: A total of 255 strains were isolated in various types of clinical specimens from 187 burn patients, of which 47.5\% were ESKAPE pathogens (121/255). Among these, MDRESKAPE pathogens accounted for 55\% (67/121). Additionally, aph3'III, mecA, bla $a_{\mathrm{SHV}}$, bla $a_{\mathrm{TEM}}, b l a_{\mathrm{PDC}}$, and bla $a_{\mathrm{SHV}}$ were the most prevalent genes detected in Enterococcus faecalis, Staphylococcus aureus, Klebsiella pneumoniae, Acinetobacter baumannii, Pseudomonas aeruginosa, and Enterobacter spp., respectively. The independent risk factors for ESKAPE infection were total body surface area (TBSA) $>30-50 \%$ (odds ratio $[\mathrm{OR}]=10.428 ; 95 \%$ confidence interval $[\mathrm{CI}], 2.047$ to 53.108$)$, TBSA $>50 \%(\mathrm{OR}=15.534 ; 95 \% \mathrm{CI}, 1.489$ to $162.021)$, and parenteral nutrition $(\mathrm{OR}=3.597 ; 95 \% \mathrm{CI}, 1.098$ to 11.787$)$. No independent risk factors were found for MDR-ESKAPE infection.

Conclusion: Clinical staff should be alert to the risk of nosocomial infection with ESKAPE pathogens in burn patients receiving parenteral nutrition and under TBSA $>30 \%$. Full attention should also be paid to the ESKAPE resistance, strict adherence to infection control protocols for the rational use of antimicrobial agents, and enhanced clinical standardization of antimicrobial agents management.

Keywords: burn, ESKAPE pathogens infection, antimicrobial resistance, risk factors

\section{Introduction}

Burn wounds are defined as skin injures and tissue damage caused by excessive heat, electricity, chemicals, and other factors; ${ }^{1}$ which have become one of the serious health problems globally. ${ }^{2}$ According to the latest report from the World Health Organization (WHO), burns cause an estimated 18,000 deaths worldwide each year. ${ }^{3}$ In addition, burn injuries are usually accompanied by many complications, and one of the most severe and common complications is microbial infection. ${ }^{4}$ Burn patients become susceptible to bacterial infections due to disrupted skin barriers and weakened innate immunity. ${ }^{5}$ Without timely and appropriate 
treatment, bacterial infections in burn patients may lead to sepsis, multiple organ dysfunction syndrome, or even death. ${ }^{6}$ A survey showed that up to $75 \%$ mortality in burn patients was due to severe burns of $40 \%$ total body surface area (TBSA) or above. ${ }^{5}$

Among burn patients, Enterococcus faecalis, Staphylococcus aureus, Klebsiella pneumoniae, Acinetobacter baumannii, Pseudomonas aeruginosa, and Enterobacter spp. are considered as important opportunistic bacteria causing infection. ${ }^{7}$ The Infectious Disease Society of America has designated these six bacterial species as "ESKAPE pathogens". ESKAPE pathogens can escape from the biocidal action of antimicrobial agents through genetic mutations and acquisition of mobile genetic elements, and mutually represent new paradigms in pathogenesis, antimicrobial resistance, and transmission. ${ }^{9}$ ESKAPE pathogens have frequently developed resistance to a variety of antimicrobial agents, such as oxazolidinones, lipopeptides, macrolides, fluoroquinolones, tetracyclines, $\beta$ lactams, and even the last resort carbapenems. ${ }^{10}$

The China Antimicrobial Surveillance Network (CHINET) reported that ESKAPE pathogens accounted for more than $50 \%$ of all clinical isolated pathogens. ${ }^{11}$ Antimicrobial-resistant ESKAPE pathogens have been considered as the global major healthcare problem by many scientists and governments. ${ }^{12}$ Moreover, the emergence of multi-drug resistant ESKAPE (MDR-ESKAPE) makes the treatment of burn patients more difficult, resulting in poor prognosis and high mortality. Therefore, appropriate control measures are highly needed to prevent bacterial infections in burn patients.

So far, no study was specifically designed to identify infectious characteristics among burn patients caused by ESKAPE pathogens. This current work analyzed the clinical manifestations, antimicrobial resistance, molecular characteristics, and risk factors for ESKAPE infection in a large teaching hospital located in Southwest China.

\section{Materials and Methods Study Design}

All bacterial infection samples between September 2018 and June 2021 were collected from burn patients admitted to Affiliated Hospital of Southwest Medical University (Luzhou, China), which is a 4200-bed large teaching hospital with 56 wards and approximately 2,100,000 annual admissions. A total of 187 patients were included in this study according to the following criteria: (1) being admitted to the hospital for treatment within $48 \mathrm{~h}$ after the burn injury; (2) matching the first six and the last of the 12 criteria for burn injury diagnosis in the Diagnostic Criteria and Treatment Guideline for Infection of Burns (2012 edition); and (3) having complete clinical information. At the same time, some cases were ruled out on the basis of the following criteria: (1) patients with immunodeficiency; (2) being admitted to hospital for treatment after $48 \mathrm{~h}$ of burns; and (3) having incomplete or missing medical record information.

\section{Data Collection and Definitions}

The samples from burn patients with bacterial infections were cultured in the standard media. Then, clinical isolates were identified using a MicroScan WalkAway 96 Plus System (Siemens, Germany) and a Microflex LT (Bruker Diagnostics Inc., USA) matrix-assisted laser desorption/ ionization time-of-flight (MALDI-TOF) mass spectrometry (MS) system. All the eligible clinical data of burn patients were collected from medical records, including basic demographics (age, gender, length of hospitalization stay, etc.), burn characteristics (cause of burn, burn depth, TBSA, inhalation injury, etc.), underlying diseases (diabetes, hypertension, abnormal liver function, hypoproteinemia, etc.), clinical treatments (surgeries, blood transfusion, parenteral nutrition, antimicrobial treatment, urethral catheterization, deep artery puncture, duration of antimicrobial application, etc.), and laboratory records (white blood cell count [WBC], percentage of neutrophils [NEU\%], hemoglobin [Hb], platelets [PLT], albuminglobulin ratio $[\mathrm{A} / \mathrm{G}$ ratio $]$ ).

\section{Antimicrobial Susceptibility Testing}

The antimicrobial susceptibility testing of the clinical bacterial isolates was performed using the VITEK system and the Kirby-Bauer disk diffusion method. Gram-positive bacteria were tested for susceptibility to 16 antibiotics, including penicillin, ampicillin, oxacillin, ciprofloxacin, levofloxacin, daptomycin, moxifloxacin, gentamicin, erythromycin, tetracycline, chloramphenicol, nitrofurantoin, vancomycin, rifampin, quinupristin-dalfopristin, and linezolid.

For Gram-negative bacteria, 17 antibiotics were selected for testing, including imipenem, meropenem, gentamicin, tobramycin, amikacin, piperacillin-tazobactam, ticarcillin-clavulanic acid, ampicillin-sulbactam, cefuroxime, cefotaxime, ceftriaxone, ceftazidime, cefepime, 
aztreonam, tetracycline, levofloxacin, and chloramphenicol.

The results were interpreted according to the Clinical and Laboratory Standards Institute (CLSI 2020). At the same time, E. faecalis ATCC29212, S. aureus ATCC25923, K. pneumoniae ATCC700603, A. baumannii ATCC19606, and $P$. aeruginosa ATCC27853 were used as quality control strains, and these strains were purchased from the Clinical Inspection Center of the Ministry of Health.

\section{Detection of Resistance Genes by Polymerase Chain Reaction}

A total of 67 MDR- ESKAPE strains were included in this experiment, including $2 \mathrm{E}$. faecalis strains, $25 \mathrm{~S}$. aureus strains, $3 \mathrm{~K}$. pneumoniae strains, 26 A. baumannii strains, 7 P. aeruginosa strains, and 4 Enterobacter spp. DNA templates were obtained by boiling bacteria, and antimicrobial resistance genes were detected by polymerase chain reaction (PCR). ${ }^{13,14}$ The PCR conditions were as described previously. These common antimicrobial resistance genes were selected based on antimicrobial susceptibility profile of each ESKAPE pathogen: E. faecium (tetM, aacA-aphD, aphA3, ermB, and aph3'III), S. aureus (mecA, tetM, ermA, qacA/B, and aph3'III), A. baumannii (bla $a_{\mathrm{TEM}}, b l a_{\mathrm{OXA}-23}, b l a_{\mathrm{OXA}-51}, b l a_{\mathrm{ADC}}$, and $\left.a d e B\right)$, $K$. pneumoniae and Enterobacter spp. (bla ${ }_{\mathrm{KPC}-2}$, bla $\mathrm{NDM}-1_{1}, \quad b l a_{\mathrm{SHV}}, \quad b l a_{\mathrm{VIM}}, \quad$ and $\left.b l a_{\mathrm{OXA}-48}\right)$, and P. aeruginosa (bla $a_{\mathrm{PDC}}, b l a_{\mathrm{OXA}-50}, b l a_{\mathrm{KPC}}, r m t B$, and $a a c$ $\left.\left(6^{\prime}\right) I b-c r\right)$. All experiment were performed in triplicated. The primers are shown in Table S1.

\section{Statistical Analysis}

Statistical analysis was performed using IBM SPSS software version 26 for Windows (IBM, NY, USA). Chisquare test, Fisher's exact test, or Mann-Whitney $U$-test were used to analyze categorical variables. Continuous variables were presented as means \pm standard deviation (SD) or as medians and interquartile range (IQR), and analyzed by the more appropriate Student $t$-test or Mann-Whitney $U$-test. Additionally, the multivariable logistic regression analysis was used to identify the independent risk factors among burn patients with ESKAPE and non-ESKAPE pathogens infection, as well as among MDR-ESKAPE and non-MDR-ESKAPE pathogens infection. Significance was defined as a $P$-value of $<0.05$ (two-tailed).

\section{Results}

\section{Distribution Characteristics of ESKAPE Pathogens}

A total of 255 isolates were collected from a variety of clinical specimens from 187 burn patients between August 2018 and June 2021. Of all the clinical specimens, wound secretions were the most frequent sample type, followed by catheter tips, blood, sputum, and others (Figure 1A). Among 187 burn patients, 46 (24.6\%) patients isolated two or more pathogens during hospitalization. Moreover, a total of 255 strains were isolated among 49 species, of which 133 (52.2\%) were Gramnegative bacteria and the other 122 (47.8\%) were Grampositive bacteria. The top 5 Gram-negative bacteria were A. baumannii (21.1\%, 28/133), P. aeruginosa (17.3\%, 23/ 133), Enterobacter spp. (17.3\%, 23/133), Escherichia coli $(8.3 \%, 11 / 133)$, and $K$. pneumoniae $(6.0 \%, 8 / 133)$ (Figure 1B). In addition, the top 3 Gram-positive bacteria were S. aureus $(28.7 \%, 35 / 122)$, Staphylococcus haemolyticus $(20.5 \%, 25 / 122)$, and Staphylococcus epidermidis (16.4\%, 20/122) (Figure 1C). Remarkably, 121 out of $255(47.5 \%)$ strains were ESKAPE pathogens, which were $S$. aureus $(28.9 \%, 35 / 121)$, A. baumannii $(23.2 \%$, 28/121), P. aeruginosa (19.0\%, 23/121), Enterobacter spp. (19.0\%, 23/121), K. pneumoniae (6.6\%, 8/121), and E. faecium $(3.3 \%, 4 / 121)$ (Figure 1D).

\section{Antimicrobial Susceptibility Profiles}

All ESKAPE isolates were subjected to antimicrobial susceptibility testing. The results are shown in Tables 1 and 2 . Only four E. faecium strains were isolated, and they had a high rate of resistance to erythromycin, oxacillin, and ciprofloxacin. $S$. aureus was highly resistant to ampicillin and penicillin, with a resistance rate of $100.0 \%$ and $97.1 \%$, respectively. However, $S$. aureus was sensitive to daptomycin, nitrofurantoin, vancomycin and linezolid. It was worth noting that 18 methicillin-resistant $S$. aureus (MRSA) isolates were detected in this study, accounting for $51.4 \%$.

No antimicrobial resistance exceeded $40 \%$ in $K$. pneumoniae except tetracycline and chloramphenicol. Moreover, more than $60 \% \mathrm{~A}$. baumannii were resistant to all tested antibiotics, and 92.9\% (26/28) MDRA. baumannii was found. In contrast, $P$. aeruginosa and Enterobacter spp. possessed the relatively lowest antimicrobial-resistant proportion. 

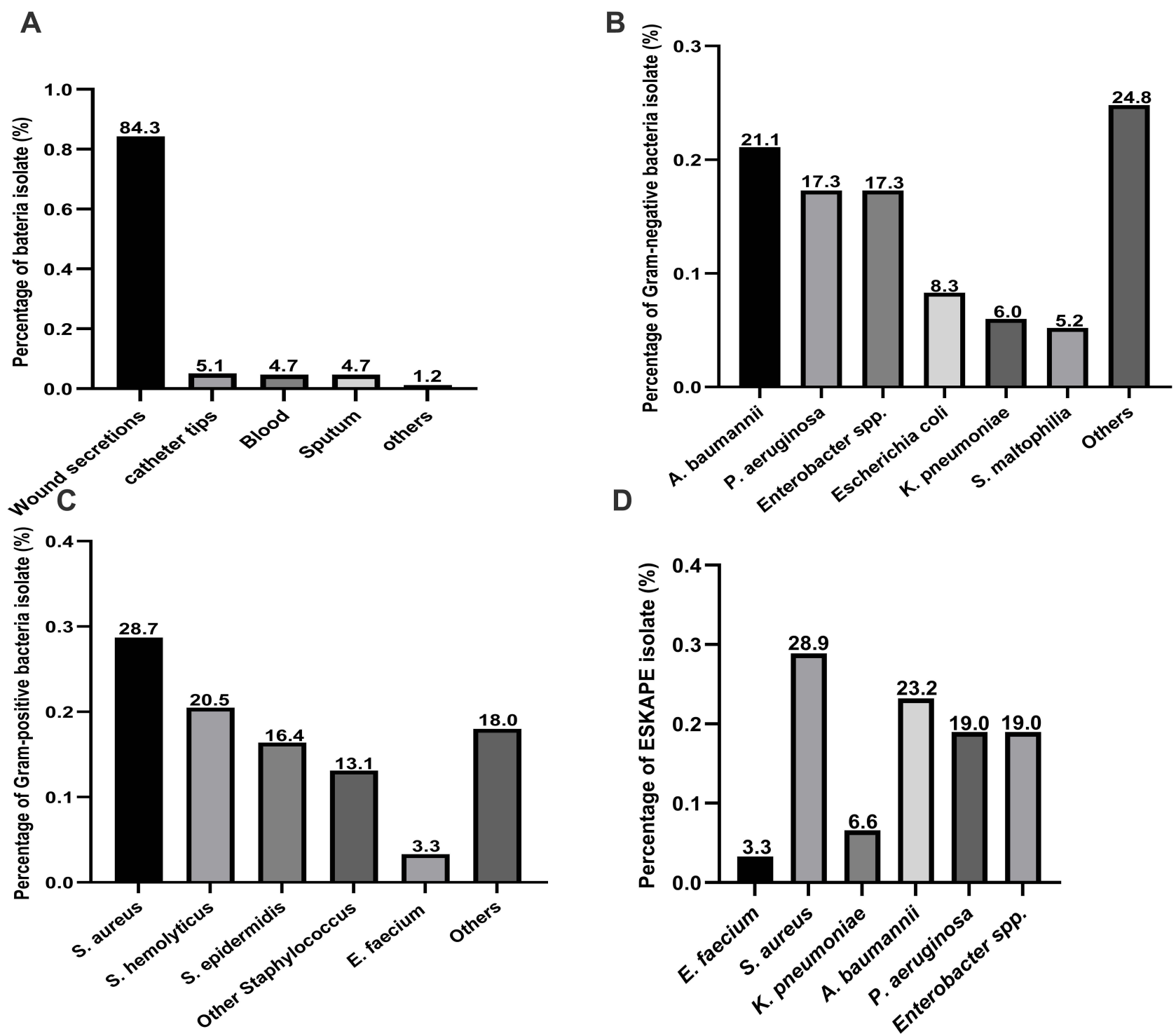

Figure I Distribution characteristics of pathogenic bacteria. (A) Strain source distribution; (B) Distribution of various types of Gram-negative bacteria; (C) Distribution of various types of Gram-positive bacteria; (D) Distribution of various types of ESKAPE pathogens.

\section{Detection of Antimicrobial Resistance}

\section{Genes}

As shown in Figure 2, only two MDR-E. faecalis isolates were found in this study, both of them contained aacA-aphD and aph3'III, and either of them carried aphA3, ermB or tetM. Among S. aureus strains, the prevalence of mecA, aph3'III, and tetM was $68.0 \%$ (17/ $25), 56.0 \%(14 / 25)$, and $52.0 \%(13 / 25)$, respectively. However, no strains expressed ermA and $q a c A / B$ genes. In $K$. pneumoniae isolates, the most prevalent gene was bla $_{\mathrm{SHV}}(100 \%, 3 / 3)$, followed by bla $a_{\mathrm{KPC}-2}(66.7 \%, 2 / 3)$ and $b l a_{\mathrm{NDM}-1}(33.3 \%, 1 / 3)$. In addition, all the A. baumannii isolates carried bla $a_{\mathrm{TEM}}$; however, none of them carried $b l a_{\mathrm{ADC}}$. At the same time, the prevalence of bla $_{\text {OXA-51 }}$, adeB, and bla ${ }_{\mathrm{OXA}-23}$ was $80.8 \%(21 / 26)$, $76.9 \%(20 / 26)$, and $53.8 \%(14 / 26)$, respectively. All the P. aeruginosa isolates carried bla $a_{\mathrm{PDC}}$, and $71.4 \%(5 / 7)$ isolates harbored bla OXA-50. Furthermore, Enterobacter spp. only expressed $b l a_{\mathrm{SHV}}(100 \%, 4 / 4)$ and $b l a_{\mathrm{OXA} 48}$ $(66.7 \%, 3 / 4)$.

\section{Analysis of ESKAPE Group versus Non-ESKAPE Group}

The potential factors among patients with ESKAPE infections are shown in Table 3. TBSA was highly associated with ESKAPE pathogens infection $(P<0.001)$. Additionally, compared with the non-ESKAPE group, more patients were critically ill $(52.2 \%$ vs $22.7 \%$; $P<$ 
Table I Antimicrobial Resistance of ESKAPE: Gram-Positive Pathogens

\begin{tabular}{|l|l|l|}
\hline Antimicrobial Agents & $\begin{array}{l}\text { S. aureus, } \mathbf{n}(\%) \\
\text { (n=35) }\end{array}$ & $\begin{array}{l}\text { E. faecium, } \mathbf{n ( \% ) ~} \\
\text { (n=4) }\end{array}$ \\
\hline Penicillin & $34(97.1)$ & $I(25.0)$ \\
Ampicillin & $35(100.0)$ & $0(0.0)$ \\
Oxacillin & $18(51.4)$ & $4(100.0)$ \\
Ciprofloxacin & $9(25.7)$ & $3(75.0)$ \\
Levofloxacin & $9(25.7)$ & $1(25.0)$ \\
Moxifloxacin & $3(8.6)$ & $1(25.0)$ \\
Daptomycin & $0(0.0)$ & $0(0.0)$ \\
Gentamicin & $8(22.9)$ & $*$ \\
Erythromycin & $25(71.4)$ & $4(100.0)$ \\
Tetracycline & $16(45.7)$ & $1(25.0)$ \\
Chloramphenicol & $22(62.9)$ & $0(0.0)$ \\
Nitrofurantoin & $0(0.0)$ & $0(0.0)$ \\
Vancomycin & $0(0.0)$ & $0(0.0)$ \\
Rifampin & $2(5.7)$ & $2(50.0)$ \\
Quinupristin-dalfopristin & $2(5.7)$ & $*$ \\
Linezolid & $0(0.0)$ & $0(0.0)$ \\
\hline
\end{tabular}

Note: *Represents that the bacterial species are naturally resistant to the antibiotic and is not included in the resistance analysis.

$0.001)$ and received a combination of antibiotics therapy (18.9\% vs $5.2 \% ; P=0.004)$, blood transfusion $(57.8 \%$ vs $26.8 \% ; P<0.001)$, parenteral nutrition $(23.3 \%$ vs $5.2 \%$; $P<0.001)$, and urethral catheterization $(33.3 \%$ vs $14.4 \%$;
$P=0.002)$. Moreover, the length of hospital stay was significantly longer in patients with ESKAPE infections $(P=0.014)$. In contrast, the value of $\mathrm{Hb}$ was lower in the non-ESKAPE group than in the ESKAPE group $(P=$ 0.034).

The multivariate logistic regression analysis showed that $\mathrm{TBSA}>30-50 \%(\mathrm{OR}=10.428 ; 95 \% \mathrm{CI}, 2.047$ to $53.108)$, TBSA $>50 \%(\mathrm{OR}=15.534 ; 95 \% \mathrm{CI}, 1.489$ to 162.021), and parenteral nutrition $(\mathrm{OR}=3.597$; 95\% CI, 1.098 to 11.787) were independent risk factors for ESKAPE pathogens infection (Table 4).

\section{Analysis of MDR-ESKAPE Group versus Non-MDR-ESKAPE Group}

The cause of burn injuries was significantly associated with MDR-ESKAPE infection $(P=0.011)$. Patients with MDR-ESKAPE infections were more likely to accept treatments such as surgery $(34.0 \%$ vs $7.9 \% ; P=0.004)$, deep artery puncture $(22.0 \%$ vs $2.6 \% ; P=0.021)$, and percutaneous tracheotomy $(20.0 \%$ vs $2.6 \% ; P=0.034)$. Additionally, patients with inhalation injury $(30.0 \%$ vs $7.9 \% ; P=0.011$ ) were also prone to MDR-ESKAPE infections (Table 5). However, no independent risk factors were found for MDR-ESKAPE infection (Table 6).

Table 2 Antimicrobial Resistance of ESKAPE: Gram-Negative Pathogens

\begin{tabular}{|c|c|c|c|c|}
\hline Antimicrobial Agents & $\begin{array}{l}\text { A. baumannii, } n(\%) \\
(n=28)\end{array}$ & $\begin{array}{l}\text { P. aeruginosa, } \mathrm{n}(\%) \\
(\mathrm{n}=23)\end{array}$ & $\begin{array}{l}\text { K. pneumoniae, } n(\%) \\
(n=8)\end{array}$ & $\begin{array}{l}\text { Enterobacter spp., } n(\%) \\
(n=23)\end{array}$ \\
\hline Imipenem & $21(75.0)$ & $3(13.1)$ & $2(25.0)$ & $3(13.1)$ \\
\hline Meropenem & $22(78.6)$ & $0(0.0)$ & $2(25.0)$ & $3(13.1)$ \\
\hline Gentamicin & $19(67.9)$ & I (4.3) & $2(25.0)$ & $2(8.7)$ \\
\hline Tobramycin & $19(67.9)$ & I (4.3) & $3(37.5)$ & $0(0.0)$ \\
\hline Amikacin & $19(67.9)$ & $0(0.0)$ & $0(0.0)$ & $0(0.0)$ \\
\hline Piperacillin-tazobactam & $23(82.1)$ & $2(8.7)$ & $0(0.0)$ & $0(0.0)$ \\
\hline Ticarcillin-clavulanic acid & $21(75.0)$ & $2(8.7)$ & $2(25.0)$ & $0(0.0)$ \\
\hline Ampicillin-Sulbactam & $23(82.1)$ & $*$ & $3(37.5)$ & $*$ \\
\hline Cefuroxime & $*$ & $*$ & $3(37.5)$ & $6(26.1)$ \\
\hline Cefotaxime & $23(82.1)$ & $*$ & $2(25.0)$ & I (4.3) \\
\hline Ceftriaxone & $23(82.1)$ & $*$ & $3(37.5)$ & $6(26.1)$ \\
\hline Ceftazidime & $23(82.1)$ & $\mathrm{I}(4.3)$ & $2(25.0)$ & I (4.3) \\
\hline Cefepime & $22(78.6)$ & $0(0.0)$ & $2(25.0)$ & $0(0.0)$ \\
\hline Aztreonam & $*$ & $4(17.4)$ & $2(25.0)$ & $0(0.0)$ \\
\hline Tetracycline & $21(75.0)$ & $*$ & $4(66.7)$ & I (4.3) \\
\hline Levofloxacin & $23(82.1)$ & $2(8.7)$ & $2(25.0)$ & $0(0.0)$ \\
\hline Chloramphenicol & $*$ & $*$ & $4(66.7)$ & $0(0.0)$ \\
\hline
\end{tabular}

Note: *Represents that the bacterial species are naturally resistant to the antibiotic and is not included in the resistance analysis. 

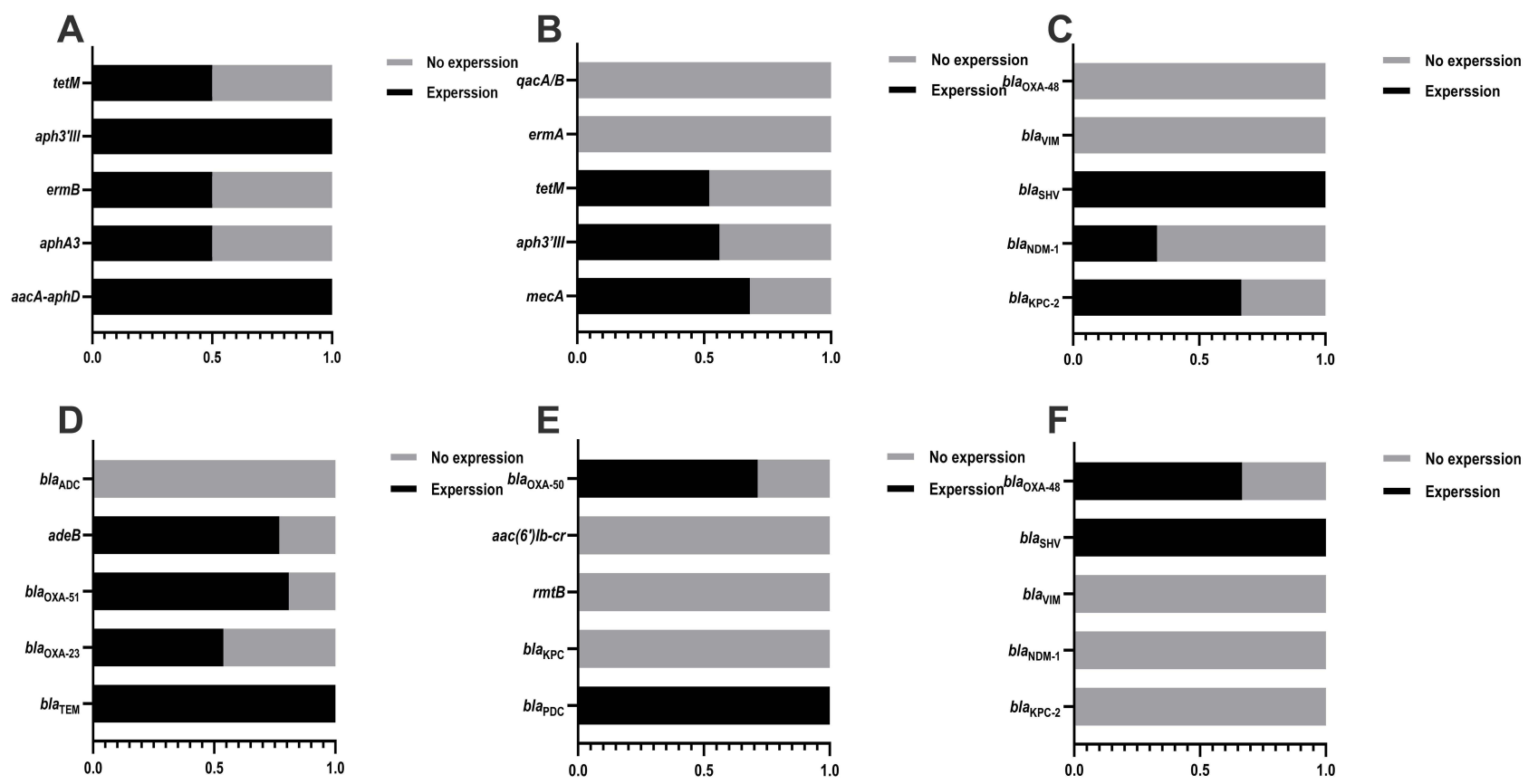

Figure 2 Expression of resistance genes in ESKAPE pathogens. (A) Expression of resistance genes in E. faecalis; (B) Expression of resistance genes in S. aureus; (C) Expression of resistance genes in K. pneumoniae; (D) Expression of resistance genes in A. baumannii; (E) Expression of resistance genes in P. aeruginosa; (F) Expression of resistance genes in Enterobacter spp.

\section{Discussion}

Burn injury is one of the most common types of traumatic injuries, with a morbidity of up to 1.1 per 100,000 population. ${ }^{5}$ The primary factors for bacterial infections in burn patients are destruction of the skin barrier and concomitant suppression of immune responses. ${ }^{15}$ In addition, the burn wound surface consists of avascular necrotic tissue, and can also provide a protein-rich micro-environment for bacterial growth. ${ }^{16}$ Since the skin barrier is damaged, staphylococci, which are originally located in sweat glands and other places, can grow in large numbers in a short time. At the same time, the wound exposed to air increases the risk of nosocomial infection. ${ }^{5}$ A total of 187 burn patients with bacterial infections from the Department of Burn and Plastic Surgery were included in this study, and 255 strains were isolated from them. Further, $84.3 \%$ strains were collected from wound secretion, which were similar to the findings of Chen's study. ${ }^{17}$ Therefore, increasing efforts to control trauma infections in burn patients was most important.

In our study, the proportion of Gram-negative bacteria was slightly higher than that of Gram-positive bacteria. This distribution was also consistent with that in other hospitals in Southwest China, ${ }^{18}$ Iran, ${ }^{19}$ and the United Kingdom. ${ }^{20}$ According to CHINET reports, S. aureus was the most susceptible bacteria in burn patients in
China, which was consistent with our results. ${ }^{11}$ S. aureus commonly colonizes human skin and proliferates when the wound is infected. ${ }^{15}$ In addition, the antimicrobialresistant $S$. aureus poses a serious threat to the treatment of post-burn bacterial infections. Here, $S$. aureus was highly resistant to $\beta$-lactam antibiotics; besides, more than half of the strains were MRSA. The detection rate of MDR-S. aureus carrying the mecA gene (resistance to many $\beta$-lactam antibiotics) reached $85 \%$, and such a high detection rate was consistent with what is now being reported internationally. ${ }^{21-23}$

A. baumannii was the most prevalent and resistant bacteria among Gram-negative bacteria. Almost all the strains were MDR-A. baumannii, and this result was also in line with some previous studies. ${ }^{18,24,25}$ A. baumannii was highly resistant to $\beta$-lactam antibiotics due to frequent expression of the extended-spectrum $\beta$-lactamases (ESBLs) encoding gene $b l a_{\mathrm{TEM}}$ and the efflux pump mediating gene $a d e B .{ }^{24,25}$ In addition, the WHO has created a priority list of antibiotic-resistant bacteria to support effective treatment research, and the carbapenem-resistant A. baumannii was on the top. ${ }^{26}$ Carbapenem resistance in A. baumannii was mainly associated with OXA-type carbapenemases, and the high carrying rates of $b l a_{\text {OXA-51 }}$ and $b l a_{\text {OXA-23 }}$ were the essential factor of specific resistance. $^{27-29}$ Importantly, these traditional antibiotics 
Table 3 Clinical Characteristics and Laboratory Records of Patients with ESKAPE Pathogens Infection

\begin{tabular}{|c|c|c|c|}
\hline Variable, n(\%) & ESKAPE $(n=90)$ & Non-ESKAPE $(n=98)$ & $P$-value \\
\hline \multicolumn{4}{|l|}{ Basic demographics } \\
\hline Age, median (range) & $5 I(4 I-7 I)$ & $50(39-63)$ & 0.826 \\
\hline Gender (male) & $55(6 I .1)$ & $61(62.8)$ & 0.803 \\
\hline Length of hospital stay, median (range), days & $31(11-46)$ & $19(9-31)$ & 0.014 \\
\hline \multicolumn{4}{|l|}{ Burn characteristics } \\
\hline Cause of burns injuries & & & 0.974 \\
\hline Flame burns & $22(24.4)$ & $51(52.5)$ & \\
\hline Hydrothermal burns & $62(68.9)$ & $38(39.2)$ & \\
\hline Others & $6(6.7)$ & $9(9.3)$ & \\
\hline Depth of burn & & & 0.900 \\
\hline Degree I-II, superficial & $40(44.4)$ & $44(45.4)$ & \\
\hline Degree II-III, deep & $50(55.6)$ & $53(54.6)$ & \\
\hline TBSA & & & $<0.001$ \\
\hline$\leq 10 \%$ & $20(22.2)$ & $45(46.4)$ & \\
\hline$>10-30 \%$ & $38(42.2)$ & $46(47.4)$ & \\
\hline$>\mathbf{3 0 - 5 0 \%}$ & $19(21.2)$ & $4(4.1)$ & \\
\hline$>\mathbf{5 0} \%$ & $13(14.4)$ & $2(2.1)$ & \\
\hline Inhalation injury & $15(16.7)$ & $12(12.4)$ & 0.404 \\
\hline \multicolumn{4}{|l|}{ Underlying conditions } \\
\hline Diabetes & $3(3.3)$ & $4(4.1)$ & 1.000 \\
\hline Hypertension & $3(3.3)$ & $7(7.2)$ & 0.393 \\
\hline Abnormal liver function & $10(11.1)$ & $6(6.2)$ & 0.229 \\
\hline Hypoproteinemia & $13(14.4)$ & $7(7.2)$ & 0.110 \\
\hline \multicolumn{4}{|l|}{ Therapy } \\
\hline Combination of antibiotics & $17(18.9)$ & $5(5.2)$ & 0.004 \\
\hline Duration of antibiotic application, median (range) & $12(6-24)$ & $7(5-12)$ & 0.052 \\
\hline Blood Transfusion & $52(57.8)$ & $26(26.8)$ & $<0.001$ \\
\hline Surgery & $20(22.2)$ & $10(10.3)$ & 0.027 \\
\hline Negative pressure suction & $8(8.9)$ & $6(6.2)$ & 0.483 \\
\hline ICU admission & $5(5.6)$ & $\mathrm{I}(\mathrm{I} .0)$ & 0.181 \\
\hline Parenteral nutrition & $21(23.3)$ & $5(5.2)$ & $<0.001$ \\
\hline Urethral catheterization & $30(33.3)$ & $14(14.4)$ & 0.002 \\
\hline Deep arterial puncture placement & $\mathrm{II}(12.2)$ & $7(3.1)$ & 0.246 \\
\hline Percutaneous tracheotomy & $10(1 \mathrm{I} . \mathrm{I})$ & $4(4.1)$ & 0.125 \\
\hline \multicolumn{4}{|l|}{ Laboratory records } \\
\hline WBC, median (range) & $12.68(8.93-18.56)$ & $9.05(7.13-12.68)$ & 0.054 \\
\hline NEU, median (range) & $82.00(73.04-87.10)$ & $70.50(59.50-81.70)$ & 0.111 \\
\hline Hb, median (range) & $129.00(109.00-143.00)$ & $135.00(117.00-151.00)$ & 0.034 \\
\hline PLT, median (range) & $189.00(116.00-256.00)$ & $211.50(160.00-167.00)$ & 0.995 \\
\hline A/G, median (range) & $1.42(1.26-1.69)$ & $1.45(1.27-1.70)$ & 0.218 \\
\hline \multicolumn{4}{|l|}{ Other characteristics } \\
\hline Malnutrition & $10(11.1)$ & $7(7.2)$ & 0.355 \\
\hline Sepsis & $6(6.7)$ & $2(2.1)$ & 0.120 \\
\hline Critically ill & $47(52.2)$ & $22(22.7)$ & $<0.001$ \\
\hline
\end{tabular}

Note: Bold indicates $P<0.05$.

Abbreviations: TBSA, total burn surface area; NEU, Neutrophil; Hb, Hemoglobin; PLT, Platelet; A/G, Albumin-globulin ratio. 
Table 4 Clinical Risk Factors for ESKAPE Infection

\begin{tabular}{|c|c|c|c|c|}
\hline \multirow[t]{2}{*}{ Variables } & \multicolumn{2}{|l|}{ Univariate Analysis } & \multicolumn{2}{|l|}{ Multivariate Analysis } \\
\hline & OR $(95 \% \mathrm{Cl})$ & $P$-value & OR $(95 \% \mathrm{CI})$ & $P$-value \\
\hline Length of hospital stay, median (range), days & $1.026(1.008-1.044)$ & 0.004 & $1.007(0.987-1.028)$ & 0.472 \\
\hline TBSA & & $<0.001$ & & 0.016 \\
\hline$\leq 10 \%$ & $\mathbf{I}$ & $<0.001$ & & 1.000 \\
\hline$>10-30 \%$ & $1.957(0.995-3.847)$ & 0.052 & $1.677(0.778-3.613)$ & 0.187 \\
\hline$>30-50 \%$ & $10.687(3.219-35.484)$ & $<0.001$ & $10.428(2.047-53.108)$ & 0.005 \\
\hline$>\mathbf{5 0} \%$ & $12.375(2.508-61.052)$ & 0.002 & $15.534(1.489-162.021)$ & 0.022 \\
\hline Combination of antibiotics & $4.285(1.509-12.164)$ & 0.006 & $2.216(0.523-9.394)$ & 0.280 \\
\hline Blood Transfusion & $3.390(1.844-6.234)$ & $<0.001$ & $0.985(0.372-2.603)$ & 0.975 \\
\hline Urethral catheterization & $2.929(1.431-5.995)$ & 0.003 & $0.628(0.210-1.880)$ & 0.406 \\
\hline Parenteral nutrition & $5.539(1.989-15.427)$ & 0.001 & 3.597 (I.098-I I.787) & 0.035 \\
\hline $\mathrm{Hb}$ & $0.986(0.973-0.999)$ & 0.036 & $0.984(0.969-1.000)$ & 0.050 \\
\hline Critically ill & $3.726(1.985-6.996)$ & $<0.001$ & $0.956(0.356-2.572)$ & 0.939 \\
\hline
\end{tabular}

Note: Bold indicates $P<0.05$.

Abbreviations: OR, odds ratio; $\mathrm{Cl}$, confidence interval.

are still widely used in clinical treatments, and such subinhibitory concentrations can also induce the antimicrobial resistance of $A$. baumannii.

$P$. aeruginosa is usually isolated from burn patients, even surpassing $S$. aureus as the most frequently detected pathogen in other previous reports, leading to a high morbidity and mortality. ${ }^{26,27}$ In the present study, $P$. aeruginosa strains were less than one fifth among ESKAPE pathogens, and the antimicrobial resistance was also relatively lower than that of other bacterial types. However, the high detection rate of $b l a_{\mathrm{PDC}}$ and $b l a_{\mathrm{OXA}-50}$ indicates that our hospital needs to be alert to the prevalence of ESBL-producing P. aeruginosa. ${ }^{30,31}$

Exposure wounds are vulnerable to infection with Enterobacter spp., especially MDR-Enterobacter spp., which was consistent with our results. ${ }^{32,33 ;}$ Moreover, burn patients with MDR-Enterobacter spp. infections may develop sepsis, multi-organ failure, and even death owing to inappropriate empirical antibiotic therapy. The high detection rate of carbapenem-resistant Enterobacter spp. reminded us that the judicious use of carbapenems is necessary. ${ }^{34}$

In addition, K. pneumoniae and E. faecalis were rarely detected in our study. It was likely that most of the strains isolated from wound secretions, but these two bacteria were usually colonized in the gastrointestinal and respiratory tracts. The high detection rate of $b l a_{\mathrm{KPC}}$ and $b l a_{\mathrm{SHV}}$ in
K. pneumoniae strains indicates that some measures should be taken to avoid the further prevalence of carbapenemresistant $K$. pneumoniae (CRKP) in our hospital. ${ }^{35,36}$ At the same time, aminoglycoside resistance genes (aacA-aphD, aphA3, aph3'III) and macrolide resistance gene (ermB) were detected in E. faecalis, which was consistent with the result of antimicrobial susceptibility profiles. ${ }^{37}$ Francisco et al have reported that ESKAPE pathogens have a significant influence on death during hospitalization in patients with severe infections. ${ }^{38}$ To further clarify the infectious characteristics of ESKAPE pathogens in burn patients, the risk factors among ESKAPE and non-ESKAPE infections as well as MDR-ESKAPE and non-MDR-ESKAPE infections were evaluated. TBSA $>30-50 \%$, TBSA $>50 \%$, and parenteral nutrition were independent risk factors for ESKAPE pathogens infection in burn patients. TBSA, as an index to assess the degree of burn injury, has been reported as an independent risk factor for burn infection. ${ }^{39}$ The larger the burn area, the more severely the skin barrier is damaged and the more the susceptibility to bacterial infections. Even commensals on the skin (such as $S$. aureus) can become a threat to cause infection on burn areas. Moreover, inevitable contact between the wound and bacteria in the air, medical devices, or the hands of medical staff is highly susceptible to nosocomial infections. Additionally, burn patients with TBSA $>20 \%$ cannot get enough nutrition independently; therefore, they have to accept parenteral nutrition. ${ }^{40}$ Although 
Table 5 Clinical Characteristics and Laboratory Records of Patients with MDR-ESKAPE Pathogens Infection

\begin{tabular}{|c|c|c|c|}
\hline Variable, n(\%) & MDR-ESKAPE $(n=50)$ & Non-MDR-ESKAPE $(n=38)$ & $P$-value \\
\hline \multicolumn{4}{|l|}{ Basic demographics } \\
\hline Age, median (range) & $19.5(2-48)$ & $3(1-32.75)$ & 0.130 \\
\hline Gender (male) & $21(42.0)$ & $23(60.5)$ & 0.085 \\
\hline Length of hospital stay, median (range), days & $19.5(8-46)$ & $12.5(9-26.5)$ & 0.158 \\
\hline \multicolumn{4}{|l|}{ Burn characteristics } \\
\hline Cause of burn injuries & & & 0.011 \\
\hline Flame burns & $16(32.0)$ & $6(15.8)$ & \\
\hline Hydrothermal burns & $27(54.0)$ & $32(84.2)$ & \\
\hline Others & $6(12.0)$ & I (2.6) & \\
\hline Depth of burn & & & 0.260 \\
\hline Degree I-II, superficial & $19(21.1)$ & $19(50.0)$ & \\
\hline Degree II-III, deep & $31(62.0)$ & $19(50.0)$ & \\
\hline TBSA & & & 0.166 \\
\hline$\leq 10 \%$ & $16(32.0)$ & II (28.9) & \\
\hline$>10-30 \%$ & $15(30.0)$ & $21(55.3)$ & \\
\hline$>30-50 \%$ & $9(18.0)$ & $6(15.8)$ & \\
\hline$>50 \%$ & $10(20.0)$ & $0(0.0)$ & \\
\hline Inhalation injury & $15(30.0)$ & $3(7.9)$ & 0.011 \\
\hline \multicolumn{4}{|l|}{ Underlying conditions } \\
\hline Diabetes & I (2.0) & $2(5.3)$ & 0.808 \\
\hline Hypertension & I (2.0) & $3(7.9)$ & 0.425 \\
\hline Abnormal liver function & I (2.0) & $2(5.3)$ & 0.808 \\
\hline Hypoproteinemia & $8(16.0)$ & $2(5.3)$ & 0.218 \\
\hline \multicolumn{4}{|l|}{ Therapy } \\
\hline Combination of antibiotics & $16(32.0)$ & $7(18.4)$ & 0.151 \\
\hline Duration of antibiotic application, median (range) & $8(6-15)$ & $7(4.75-10.00)$ & 0.080 \\
\hline Blood Transfusion & $29(58.0)$ & $22(57.9)$ & 0.992 \\
\hline Surgery & $17(34.0)$ & $3(7.9)$ & 0.004 \\
\hline Negative pressure suction & $6(12.0)$ & $2(5.3)$ & 0.475 \\
\hline ICU admission & $5(10.0)$ & $2(5.2)$ & 0.678 \\
\hline Parenteral nutrition & $14(28.0)$ & $7(18.4)$ & 0.296 \\
\hline Urethral catheterization & $20(40.0)$ & $10(26.3)$ & 0.180 \\
\hline Deep arterial puncture placement & II (22.0) & I (2.6) & 0.021 \\
\hline Percutaneous tracheotomy & $10(20.0)$ & $I(2.6)$ & 0.034 \\
\hline \multicolumn{4}{|l|}{ Laboratory records } \\
\hline WBC, median (range) & II.74 (8.89-17.83) & $12.37(9.82-17.49)$ & 0.886 \\
\hline NEU, median (range) & $73.7(45.62-84.3)$ & $64.82(54.12-76.52)$ & 0.354 \\
\hline $\mathrm{Hb}$, median (range) & $125.00(|07.75-| 4 \mid .50)$ & II $4.00(99.00-136.00)$ & 0.067 \\
\hline PLT, median (range) & $238.5(164.25-346.75)$ & $270(210.25-344.50)$ & 0.134 \\
\hline A/G, median (range) & $1.61(1.40-1.90)$ & $1.72(1.50-2.29)$ & 0.445 \\
\hline \multicolumn{4}{|l|}{ Other characteristics } \\
\hline Malnutrition & $5(10.0)$ & $4(10.8)$ & 1.000 \\
\hline Sepsis & $5(10.0)$ & I (2.6) & 0.352 \\
\hline Critically ill & $28(56.0)$ & $18(47.4)$ & 0.557 \\
\hline
\end{tabular}

Note: Bold indicates $P<0.05$.

Abbreviations: TBSA, total burn surface area; NEU, Neutrophil; Hb, Hemoglobin; PLT, Platelet; A/G, Albumin-globulin ratio. 
Table 6 Clinical Risk Factors for MDR-ESKAPE Infection

\begin{tabular}{|c|c|c|c|c|}
\hline \multirow[t]{2}{*}{ Variables } & \multicolumn{2}{|l|}{ Univariate Analysis } & \multicolumn{2}{|l|}{ Multivariate Analysis } \\
\hline & OR $(95 \% \mathrm{CI})$ & $P$-value & OR $(95 \% \mathrm{CI})$ & $P$-value \\
\hline Cause of burn injuries & & 0.016 & & 0.450 \\
\hline Flame burns & & 0.016 & & 0.450 \\
\hline Hydrothermal burns & $0.248(0.08 I-0.76 I)$ & 0.015 & $2.67 \mid(0.209-34.100)$ & 0.450 \\
\hline Others & $1.765(0.170-18.321)$ & 0.634 & $0.485(0.116-2.026)$ & 0.321 \\
\hline Inhalation injury & $5.000(1.329-18.814)$ & 0.017 & $1.712(0.292-10.033)$ & 0.551 \\
\hline Surgery & $6.010(1.611-22.415)$ & 0.008 & $2.27 \mid(0.448-11.5 \mid 8)$ & 0.322 \\
\hline Deep arterial puncture placement & $10.436(1.283-84.877)$ & 0.028 & $2.195(0.182-26.481)$ & 0.536 \\
\hline Percutaneous tracheotomy & $9.250(1.129-75.815)$ & 0.038 & $3.068(0.267-35.205)$ & 0.368 \\
\hline
\end{tabular}

Abbreviations: $\mathrm{OR}$, odds ratio; $\mathrm{Cl}$, confidence interval.

parenteral nutrition can supply sufficient nutrition to patients, the duration of parenteral nutrition leads to longer wound exposure, which also provides more opportunities for ESKAPE infection. ${ }^{41}$ Prolonged parenteral nutrition may also cause intestinal dysbiosis. Hiengrach et al suggested that intestinal dysbiosis could, to some extent, lead to infections with $P$. aeruginosa and some Enterobacter spp. ${ }^{42}$ Remarkably, despite statistically significant differences between non-MDR-ESKAPE and MDR-ESKAPE infections in burn injuries, inhalation injury, surgery, deep artery puncture, and percutaneous tracheotomy were established, the risk factors for MDR-ESKAPE infection were not revealed. One possible explanation may be the limited number of MDR-ESKAPE infection cases in our study.

This study also had some limitations. First, it was single-center research, and some important risk factors for ESKAPE infection might have been missed. Second, sample sizes were small, leading to a selection bias in the results. Third, it was a retrospective study, and some medical records and ESKAPE isolates might not have been stored completely.

\section{Conclusion}

To our best knowledge, this study was novel in evaluating pathogenic characteristics and risk factors for ESKAPE pathogens infection in burn patients. ESKAPE pathogens account for almost $50 \%$ of all the bacteria isolated from burn patients, and carrying antimicrobial resistance genes was the main reason for these strains to be highly resistant. The severe antimicrobial resistance of ESKAPE pathogens poses great challenges in the treatment of infections and the control of nosocomial infections. TBSA $>30 \%-50 \%$, TBSA $>$ $50 \%$, and parenteral nutrition were identified as the independent risk factors for ESKAPE pathogens infection. A series of reasonable and effective measures are essential to prevent the spread of ESKAPE pathogens in hospitals, such as strengthening the hand hygiene of medical and nursing staff, strictly enforcing the aseptic operation and sterilization isolation system, and sterilizing the relevant instruments for patients who are hospitalized and receive mechanical ventilation for a long time.

\section{Abbreviations}

TBSA, total body surface area; CHINET, China Antimicrobial Surveillance Network; IDSA, Infectious Disease Society of America; MDR-ESKAPE, multi-drug resistant ESKAPE; WHO, the World Health Organization; WBC, white blood cell count; NEU\%, percentage of neutrophils; Hb, hemoglobin; PLT,platelets; A/G ratio, albumin-globulin ratio.

\section{Data Sharing Statement}

The datasets generated during and/or analyzed during the current study are available from the corresponding author on reasonable request.

\section{Ethics Approval and Consent to Participate}

This study was approved by the Institutional Review Board of affiliated hospital of southwest medical university in accordance with the Declaration of Helsinki (KY2020043). Written informed consent was obtained from all participants. 


\section{Author Contributions}

ZL and JX contributed to conceive this study, analyze the data, and write the manuscript. JH, YD and ZZ collected the data. JY, ZD, and SL performed the experiments. ZL edited and confirmed final manuscript. JL provided the financial support. All authors contributed to manuscript revision, read, and approved the submitted version.

\section{Funding}

This work was supported by the grants from Sichuan Science and Technology Program (2021YFS0329, 0QYCX0056), Southwest Medical University Science Program (2020ZRQNB025), and Luxian Government and Southwest Medical University Cooperation Program (2020LXXNYKD-04).

\section{Disclosure}

The authors declare that they have no conflicts of interest in this work.

\section{References}

1. Sadeghi-Bazargani H, Mohammadi R, Amiri S, et al. Individuallevel predictors of inpatient childhood burn injuries: a case-control study. BMC Public Health. 2016;16(1):209. doi:10.1186/s12889016-2799-1

2. Wen JJ, Cummins C, Radhakrishnan RS. Sildenafil recovers burn-induced cardiomyopathy. Cells. 2020;9(6):1393. doi:10.3390/ cells 9061393

3. Lee KC, Dretzke J, Grover L, Logan A, Moiemen N. A systematic review of objective burn scar measurements. Burns Trauma. 2016;4:14. doi:10.1186/s41038-016-0036-x

4. Lachiewicz AM, Hauck CG, Weber DJ, Cairns BA, van Duin D. Bacterial infections after burn injuries: impact of multidrug resistance. Clin Infect Dis. 2017;65(12):2130-2136. doi:10.1093/ cid/cix682

5. Church D, Elsayed S, Reid O, Winston B, Lindsay R. Burn wound infections. Clin Microbiol Rev. 2006;19(2):403-434.

6. Zhang P, Zou B, Liou YC, Huang C. The pathogenesis and diagnosis of sepsis post burn injury. Burns Trauma. 2021;9:tkaa047. doi:10.1093/burnst/tkaa047

7. Glasser JS, Landrum ML, Chung KK, et al. Description of Streptococcus pneumoniae infections in burn patients. Burns. 2010;36(4):528-532. doi:10.1016/j.burns.2009.07.006

8. Richwagen N, Lyles JT, Dale BLF, Quave CL. Antibacterial activity of Kalanchoe mortagei and K. fedtschenkoi against ESKAPE pathogens. Front Pharmacol. 2019;10:67. doi:10.3389/fphar.2019.00067

9. De Oliveira DMP, Forde BM, Kidd TJ, et al. Antimicrobial resistance in ESKAPE pathogens. Clin Microbiol Rev. 2020;33(3):e00181e00219. doi:10.1128/CMR.00181-19.

10. Peeters P, Ryan K, Karve S, et al. The impact of initial antibiotic treatment failure: real-world insights in patients with complicated, health care-associated intra-abdominal infection. Infect Drug Resist. 2019;12:329-343. doi:10.2147/IDR.S184116

11. Hu F, Guo Y, Yang Y, et al. Resistance reported from China antimicrobial surveillance network (CHINET) in 2018. Eur $J$ Clin Microbiol Infect Dis. 2019;38(12):2275-2281. doi:10.1007/s10096019-03673-1
12. Rossi R, Ciofalo M. An updated review on the synthesis and antibacterial activity of molecular hybrids and conjugates bearing Imidazole Moiety. Molecules. 2020;25(21):5133. doi:10.3390/ molecules25215133

13. Li Z, Ding Z, Liu Y, et al. Phenotypic and genotypic characteristics of biofilm formation in clinical isolates of Acinetobacter baumannii. Infect Drug Resist. 2021;14:2613-2624. doi:10.2147/IDR.S310081

14. Li Z, Ding Z, Yang J, et al. Carbapenem-resistant Klebsiella pneumoniae in southwest China: molecular characteristics and risk factors caused by KPC and NDM producers. Infect Drug Resist. 2021;14:3145-3158. doi:10.2147/IDR.S324244

15. Ladhani HA, Yowler CJ, Claridge JA. Burn wound colonization, infection, and sepsis. Surg Infect. 2021;22(1):44-48. doi:10.1089/ sur.2020.346

16. Erol S, Altoparlak U, Akcay MN, Celebi F, Parlak M. Changes of microbial flora and wound colonization in burned patients. Burns. 2004;30(4):357-361. doi:10.1016/j.burns.2003.12.013

17. Chen H, Yang L, Cheng L, Hu XH, Shen YM. Distribution and drug resistance of pathogens in burn patients in China from 2006 to 2019. World J Clin Cases. 2021;9(10):2228-2237. doi:10.12998/wjcc.v9. i10.2228

18. Gong Y, Peng Y, Luo X, et al. Different infection profiles and antimicrobial resistance patterns between burn ICU and common wards. Front Cell Infect Microbiol. 2021;11:681731. doi:10.3389/ fcimb.2021.681731

19. Hashemzadeh M, Heydari R, Asareh Zadegan Dezfuli A, Saki M, Meghdadi H, Bakhtiyariniya P. Occurrence of multiple-drug resistance bacteria and their antimicrobial resistance patterns in burn infections from southwest of Iran. J Burn Care Res. 2021. doi:10.1093/jbcr/irab097

20. Escandon-Vargas K, Tangua AR, Medina P, et al. Healthcareassociated infections in burn patients: timeline and risk factors. Burns. 2020;46(8):1775-1786. doi:10.1016/j.burns.2020.04.031

21. Karmakar A, Dua P, Ghosh C. Biochemical and molecular analysis of Staphylococcus aureus clinical isolates from hospitalized patients. Can J Infect Dis Med Microbiol. 2016;2016:9041636. doi:10.1155/ 2016/9041636

22. Karmakar A, Jana D, Dutta K, Dua P, Ghosh C. Prevalence of panton-valentine leukocidin gene among community acquired Staphylococcus aureus: a real-time PCR study. $J$ Pathog. 2018;2018:4518541. doi:10.1155/2018/4518541

23. Jahanshahi A, Zeighami H, Haghi F. Molecular characterization of methicillin and vancomycin resistant Staphylococcus aureus strains isolated from hospitalized patients. Microb Drug Resist. 2018;24 (10):1529-1536. doi:10.1089/mdr.2018.0069

24. Gong Y, Shen X, Huang G, et al. Epidemiology and resistance features of Acinetobacter baumannii isolates from the ward environment and patients in the burn ICU of a Chinese hospital. J Microbiol. 2016;54(8):551-558. doi:10.1007/s12275-016-6146-0

25. Sarhaddi N, Soleimanpour S, Farsiani H, et al. Elevated prevalence of multidrug-resistant Acinetobacter baumannii with extensive genetic diversity in the largest burn centre of northeast Iran. $J$ Glob Antimicrob Resist. 2017;8:60-66. doi:10.1016/j.jgar.2016.10.009

26. Tacconelli E, Carrara E, Savoldi A, et al. Discovery, research, and development of new antibiotics: the WHO priority list of antibiotic-resistant bacteria and tuberculosis. Lancet Infect Dis. 2018;18(3):318-327. doi:10.1016/S1473-3099(17)30753-3

27. Zhao Y, Hu K, Zhang J, et al. Outbreak of carbapenem-resistant Acinetobacter baumannii carrying the carbapenemase OXA-23 in ICU of the eastern Heilongjiang Province, China. BMC Infect Dis. 2019;19(1):452. doi:10.1186/s12879-019-4073-5

28. El Bannah AMS, Nawar NN, Hassan RMM, Salem STB. Molecular epidemiology of carbapenem-resistant Acinetobacter baumannii in a tertiary care hospital in Egypt: clonal spread of blaOXA-23. Microb Drug Resist. 2018;24(3):269-277. doi:10.1089/mdr.20 17.0057 
29. Jain M, Sharma A, Sen MK, Rani V, Gaind R, Suri JC. Phenotypic and molecular characterization of Acinetobacter baumannii isolates causing lower respiratory infections among ICU patients. Microb Pathog. 2019;128:75-81. doi:10.1016/j.micpath.2018.12.023

30. Manandhar S, Zellweger RM, Maharjan N, et al. A high prevalence of multi-drug resistant Gram-negative bacilli in a Nepali tertiary care hospital and associated widespread distribution of Extended-Spectrum Beta-Lactamase (ESBL) and carbapenemase-encoding genes. Ann Clin Microbiol Antimicrob. 2020;19(1):48. doi:10.1186/s12941-020-00390-y

31. Logan LK, Weinstein RA. The epidemiology of carbapenem-resistant Enterobacteriaceae: the impact and evolution of a global menace. $J$ Infect Dis. 2017;215(suppl_1):S28-S36. doi:10.1093/infdis/jiw282

32. Gallaher JR, Banda W, Lachiewicz AM, Krysiak R, Cairns BA, Charles AG. Colonization with multidrug-resistant Enterobacteriaceae is associated with increased mortality following burn injury in Sub-Saharan Africa. World J Surg. 2018;42 (10):3089-3096. doi:10.1007/s00268-018-4633-7

33. Mirzaei B, Babaei R, Bazgir ZN, Goli HR, Keshavarzi S, Amiri E. Prevalence of Enterobacteriaceae spp. and its multidrug-resistant rates in clinical isolates: a two-center cross-sectional study. Mol Biol Rep. 2021;48(1):665-675. doi:10.1007/s11033-020-06114-x

34. Cohen Mendel L, Amity K, Katz DE, Lazarovitch T, Zaidenstein R, Marchaim D. The epidemiology of carbapenem resistant Enterobacter spp: a case-case-control matched analysis. Infect Control Hosp Epidemiol. 2021;42(6):754-759. doi:10.1017/ice.2020.1286

35. Liao W, De Wang L, Li D, et al. High prevalence of $16 \mathrm{~s}$ rRNA methylase genes among carbapenem-resistant hypervirulent Klebsiella pneumoniae isolates in a Chinese Tertiary Hospital. Microb Drug Resist. 2021;27(1):44-52. doi:10.1089/mdr.20 19.0482
36. Yu X, Zhang W, Zhao Z, et al. Molecular characterization of carbapenem-resistant Klebsiella pneumoniae isolates with focus on antimicrobial resistance. BMC Genomics. 2019;20(1):822. doi:10.1186/ s12864-019-6225-9

37. Madaha EL, Gonsu HK, Bughe RN, Fonkoua MC, Ateba CN, Mbacham WF. Occurrence of blaTEM and blaCTXM genes and biofilm-forming ability among clinical isolates of Pseudomonas aeruginosa and Acinetobacter baumannii in Yaounde, Cameroon. Microorganisms. 2020;8(5):708. doi:10.3390/microorganisms8050708

38. Francisco J, Aragao I, Cardoso T. Risk factors for long-term mortality in patients admitted with severe infection. BMC Infect Dis. 2018;18 (1):161. doi:10.1186/s12879-018-3054-4

39. Pragasam AK, Vijayakumar S, Bakthavatchalam YD, et al. Molecular characterisation of antimicrobial resistance in Pseudomonas aeruginosa and Acinetobacter baumannii during 2014 and 2015 collected across India. Indian $J$ Med Microbiol. 2016;34(4):433-441. doi:10.4103/0255-0857.195376

40. Dylewksi ML, Baker M, Prelack K, et al. The safety and efficacy of parenteral nutrition among pediatric patients with burn injuries. Pediatr Crit Care Med. 2013;14(3):e120-e125. doi:10.1097/ PCC.0b013e3182712b2b

41. You T, Zhang H, Guo L, Ling KR, Hu XY, Li LQ. Differences in clinical characteristics of early- and late-onset neonatal sepsis caused by Klebsiella pneumoniae. Int J Immunopathol Pharmacol. 2020;34:2058738420950586. doi:10.1177/2058738420950586

42. Hiengrach P, Panpetch W, Worasilchai N, et al. Administration of candida albicans to dextran sulfate solution treated mice causes intestinal dysbiosis, Emergence and dissemination of intestinal Pseudomonas Aeruginosa and lethal sepsis. Shock. 2020;53 (2):189-198. doi:10.1097/SHK.0000000000001339
Infection and Drug Resistance

\section{Publish your work in this journal}

Infection and Drug Resistance is an international, peer-reviewed openaccess journal that focuses on the optimal treatment of infection (bacterial, fungal and viral) and the development and institution of preventive strategies to minimize the development and spread of resistance. The journal is specifically concerned with the epidemiology of antibiotic resistance and the mechanisms of resistance development and diffusion in both hospitals and the community. The manuscript management system is completely online and includes a very quick and fair peerreview system, which is all easy to use. Visit http://www.dovepress.com/ testimonials.php to read real quotes from published authors. 